\title{
The cancellation problem over Noetherian one-dimensional domains
}

\author{
M'hammed El Kahoui and Mustapha Ouali
}

\begin{abstract}
Let $R$ be a commutative Noetherian one-dimensional domain containing $\mathbb{Q}$. In this paper we prove that if an $R$-algebra $A$ is such that $A^{[n]} \cong_{R} R^{[n+2]}$, for some $n \geq 1$, then $A \cong_{R} R^{[2]}$. In terms of affine fibrations this means that every stably trivial $\mathbb{A}^{2}$-fibration over $R$ is actually trivial. On the other hand, it is known that this result does not hold in general if $R$ has dimension at least two or if $R$ does not contain $\mathbb{Q}$.
\end{abstract}

\section{Introduction}

Throughout, all rings are assumed to be commutative with unity. Given a ring $R$ and a positive integer $m$ we denote by $R^{[m]}$ the polynomial $R$-algebra in $m$ variables (by convention we let $R^{[0]}=R$ ). By a coordinate system of $R^{[m]}$ we mean a list $x=x_{1}, \ldots, x_{m}$ of $m$ polynomials which generates $R^{[m]}$ as an $R$-algebra.

Let us recall the following fundamental problem of affine algebraic geometry, known as the Zariski cancellation problem (see, e.g., [23], [21]).

\section{PROBLEM 1 (CANCELLATION PROBLEM)}

Let $K$ be a field, and let $(m, n)$ be a pair of positive integers. Given a $K$-algebra $A$ such that $A^{[n]} \cong_{K} K^{[m+n]}$, does it follow that $A \cong_{K} K^{[m]}$ ?

We will say that the cancellation property holds for $(m, n)$ if the above problem has a positive answer for every field $K$.

The fact that the cancellation property holds for $(1, n)$ follows essentially from the results of Abhyankar, Heinzer, and Eakin [1]. More generally, it follows from the results of Hamann [17] that the $(1, n)$-cancellation property still holds if instead of fields one considers Noetherian rings containing $\mathbb{Q}$. On the other hand, from the results of Miyanishi and Sugie [24], Fujita [16], and Kambayashi [19] it follows that the cancellation property holds for $(2, n)$ in the case of fields of charateristic zero. The case of algebraically closed fields of positive characteristic was proved by Russell in [25]. It was also proved by Derksen, van den Essen, and van Rossum [8] that the $(2, n)$-cancellation property holds true when fields are

Kyoto Journal of Mathematics, Vol. 54, No. 1 (2014), 157-165

DOI 10.1215/21562261-2400301, (C) 2014 by Kyoto University

Received May 15, 2012. Revised December 25, 2012. Accepted January 15, 2013.

2010 Mathematics Subject Classification: Primary 14R25; Secondary 14R10.

Authors' work partially supported by Centre National pour la Recherche Scientifíque et Techníque

(Morocco) project URAC01. 
replaced by Dedekind domains containing $\mathbb{Q}$. For $m \geq 3$, the problem is to our knowledge still open, but a candidate counterexample in positive characteristic was given by Asanuma in [2].

The main result of this paper is that the $(2, n)$-cancellation property holds over an arbitrary Noetherian one-dimensional domain $R$ containing $\mathbb{Q}$. The assumptions that $R$ is one-dimensional and contains $\mathbb{Q}$ cannot be dropped. Indeed, a classical example of Hochster [18] shows that this result does not hold in general if $R$ has dimension at least two. Another classical example of Asanuma [2, Theorem 5.1] shows that the result does not hold in general if $R$ is a one-dimensional domain which does not contain $\mathbb{Q}$.

The paper is organized as follows. In Section 2 we recall the results of affine fibrations theory to be used in this paper. Section 3 is devoted to the proof of the main result of this paper. As a consequence of the main result we show that if $A$ is an $\mathbb{A}^{2}$-fibration over $R=K^{[2]}$, where $K$ is a field of characteristic zero, then $A / p A \cong_{R / p R}(R / p R)^{[2]}$ for every prime polynomial $p \in R$. This answers in particular a question raised by Vénéreau (see [27] and [15, Problem 13]) concerning the polynomial $v_{1}=y+x\left[x z+y\left(y u+z^{2}\right)\right]$ in $\mathbb{C}[x, y, z, u]$, a candidate counterexample to several open problems in affine algebraic geometry.

\section{Affine fibrations}

In this section we recall the results of affine fibrations theory to be used in this paper. Given a ring $R$ and $\mathfrak{p} \in \operatorname{Spec} R$, the residue field $R_{\mathfrak{p}} / \mathfrak{p} R_{\mathfrak{p}}$ is denoted by $K(\mathfrak{p})$. Given an $R$-module $M$ we let $\operatorname{Sym}_{R}(M)$ be the symmetric algebra of $M$. For an $R$-algebra $A$ we let $\Omega_{A / R}$ (resp., $\left.\operatorname{Der}_{R}(A)\right)$ be the $A$-module of Kähler differentials of $A$ over $R$ (resp., $R$-derivations of $A$ ).

\section{DEFINITION 2.1}

Given $m \geq 0$, an $R$-algebra $A$ is said to be an $\mathbb{A}^{m}$-fibration over $R$ if it satisfies the following properties.

i. $\quad A$ is finitely generated as an $R$-algebra.

ii. $A$ is flat as an $R$-module.

iii. For every $\mathfrak{p} \in \operatorname{Spec} R$ we have $K(\mathfrak{p}) \otimes_{R} A \cong \cong_{K(\mathfrak{p})} K(\mathfrak{p})^{[m]}$.

From the property (iii) one easily deduces that the morphism $\operatorname{Spec} A \longrightarrow \operatorname{Spec} R$, induced by the homomorphism $R \longrightarrow A$, is surjective. This property together with the flatness assumption implies that $A$ is faithfully flat over $R$. In particular, the homomorphism $R \longrightarrow A$ is injective, and hence we can view $R$ as a subring of $A$.

An $\mathbb{A}^{m}$-fibration $A$ over $R$ is said to be trivial if $A \cong_{R} R^{[m]}$. The fibration is said to be stably trivial if $A^{[n]} \cong{ }_{R} R^{[m+n]}$ for some $n \geq 0$.

The following fundamental result due to Asanuma concerns the stable structure of $\mathbb{A}^{m}$-fibrations (see $[2$, Theorem 3.4$]$ ). 
THEOREM 2.2

Let $R$ be a Noetherian ring, and let $A$ be an $\mathbb{A}^{m}$-fibration over $R$. Then $\Omega_{A / R}$ is a finitely generated projective A-module of rank $m$. Moreover, $A$ is up to isomorphism an $R$-subalgebra of $R^{[n]}$ for some $n$ such that

$$
A^{[n]} \cong \operatorname{Sym}_{R^{[n]}}\left(R^{[n]} \otimes_{A} \Omega_{A / R}\right)
$$

as $R$-algebras.

As a direct consequence of Theorem 2.2, if an $\mathbb{A}^{m}$-fibration $A$ over a Noetherian ring $R$ is such that $\Omega_{A / R}$ is a free $A$-module, then $A$ is stably trivial. Another consequence of Theorem 2.2 (see [2, Corollary 3.5]) is that if $A$ is an $\mathbb{A}^{m}$-fibration over a regular ring $R$, then there exists $n \geq 0$ and a rank $m$ finitely generated projective $R$-module $M$ such that $A^{[n]} \cong_{R} \operatorname{Sym}_{R}(M)^{[n]}$. In particular, if $R$ is a polynomial ring over a field, then by the Quillen-Suslin theorem $A$ is stably trivial.

\subsection{A criterion for an $\mathbb{A}^{1}$-fibration to be trivial}

In this subsection we give a criterion for an $\mathbb{A}^{1}$-fibration, over an arbitrary Noetherian domain containing $\mathbb{Q}$, to be trivial. For this, we need to recall the following cancellation result due to Hamann [17].

\section{THEOREM 2.3}

Let $R$ be a Noetherian ring containing $\mathbb{Q}$. Then for every $R$-algebra $A$ such that $A^{[n]} \cong_{R} R^{[n+1]}$, for some $n \geq 1$, we have $A \cong_{R} R^{[1]}$.

Combining Theorems 2.2 and 2.3 yields the following result (see [5, Theorem 3.4]).

\section{THEOREM 2.4}

Let $R$ be a Noetherian ring containing $\mathbb{Q}$, and let $A$ be an $\mathbb{A}^{1}$-fibration over $R$. Then $A$ is trivial over $R$ if and only if $\Omega_{A / R}$ is a free $A$-module.

Recall that an $R$-derivation $\xi \in \operatorname{Der}_{R}(A)$ is said to be fixed point free if its image generates the unit ideal of $A$.

Let $A$ be an $\mathbb{A}^{m}$-fibration over $R$. By Theorem 2.2 the $A$-module $\Omega_{A / R}$ is finitely generated and projective. From the well-known fact that finitely generated projective modules are reflexive it follows that the freeness of $\Omega_{A / R}$ is equivalent to the freeness of its dual $\operatorname{Der}_{R}(A)$. As a consequence, we have the following result.

\section{COROLLARY 2.5}

Let $R$ be a Noetherian domain containing $\mathbb{Q}$, and let $A$ be an $\mathbb{A}^{1}$-fibration over $R$. Then $A$ is trivial over $R$ if and only if there exists $\xi \in \operatorname{Der}_{R}(A)$ which is fixed point free. 


\section{Proof}

Clearly, if $A$ is trivial over $R$, say, $A=R[v]=R^{[1]}$, then the $R$-derivation of $A$ defined by $\xi(v)=1$ is fixed point free. Conversely, let $\xi \in \operatorname{Der}_{R}(A)$ be fixed point free, and let $\xi_{1} \in \operatorname{Der}_{R}(A)$. Let $K$ be the quotient field of $R$, and let $S=R \backslash\{0\}$. Since $A$ is an $\mathbb{A}^{1}$-fibration over $R$ and $R$ is a domain we have $K \otimes_{R} A \cong_{K} K^{[1]}$. Since, moreover, $K \otimes_{R} A \cong_{K} A_{S}$ we have $A_{S} \cong_{K} K^{[1]}$, and hence we can find $v \in A$ transcendental over $R$ such that $A_{S}=K[v]$. Thus, if we let $\xi(v)=\alpha$ and $\xi_{1}(v)=\beta$, then $\alpha, \beta \in A$ and we have $\alpha \xi_{1}=\beta \xi$. The assumption that $\xi$ is fixed point free implies that there exist $a_{1}, \ldots, a_{r} \in A$ and $u_{1}, \ldots, u_{r} \in A$ such that $\sum u_{i} \xi\left(a_{i}\right)=1$. This yields $\alpha \sum u_{i} \xi_{1}\left(a_{i}\right)=\beta$ and hence $\xi_{1}=\beta_{1} \xi$, where $\beta_{1}=\sum u_{i} \xi_{1}\left(a_{1}\right)$. Thus, $\operatorname{Der}_{R}(A)=A \xi$, and so it is free. Since on the other hand $\Omega_{A / R}$ is reflexive and its dual $\operatorname{Der}_{R}(A)$ is free the $A$-module $\Omega_{A / R}$ is free as well. By Theorem 2.4, $A$ is trivial over $R$.

We will also need the well-known fact that every $\mathbb{A}^{1}$-fibration over a principal ideal domain (PID) is trivial. In fact, much more general results can be found in the literature (see, e.g., [20], [9], [6], [10]), but they will not be needed for our purpose.

\subsection{Some results on $\mathbb{A}^{2}$-fibrations}

A well known result due to Sathaye [26, Theorem 1] asserts that every $\mathbb{A}^{2}$ fibration over a discrete valuation ring containing $\mathbb{Q}$ is trivial. This result together with the results of Bass, Connell, and Wright [4] implies that every $\mathbb{A}^{2}$-fibration over a PID containing $\mathbb{Q}$ is trivial (see [5, Corollary 4.8]). For an arbitrary Noetherian one-dimensional domain containing $\mathbb{Q}$, Asanuma and Bhatwadekar proved in [3, Theorem 3.8] the following generalization of this result.

\section{THEOREM 2.6}

Let $R$ be a Noetherian one-dimensional domain containing $\mathbb{Q}$, and let $A$ be an $\mathbb{A}^{2}$-fibration over $R$. Then there exists $u \in A$ transcendental over $R$ such that $A$ is an $\mathbb{A}^{1}$-fibration over $R[u]$.

If in addition to the assumptions of Theorem 2.6 the ring $R$ is seminormal, then $A \cong{ }_{R} \operatorname{Sym}_{R}(M)^{[1]}$, where $M$ is a finitely generated projective $R$-module of rank one (see [3, Corollary 3.9]).

Based on the fact that the cancellation property holds for $(2, n)$ in characteristic zero, Freudenburg proved in [14, Corollary 2.2] the following result.

\section{THEOREM 2.7}

Let $R$ be a ring containing $\mathbb{Q}$, and let $A$ be an $R$-algebra such that $A^{[n]} \cong_{R} R^{[n+2]}$ for some $n \geq 1$. Then $A$ is an $\mathbb{A}^{2}$-fibration over $R$.

\section{REMARK 2.8}

In [14, Theorem 3.1], Freudenburg proved a result for $\mathbb{A}^{2}$-fibrations over polynomial rings similar to Corollary 2.5. Given a field $K$ of characteristic zero, the 
result in question states that an $\mathbb{A}^{2}$-fibration $A$ over $R=K^{[n]}$ is trivial if and only if there exists a locally nilpotent $R$-derivation $\xi$ of $A$ with a slice. Then in $[14$, Question 2], the author asks whether the condition that $\xi$ has a slice can be weakened to the condition that $\xi$ is fixed point free. In a recent paper [11], we proved that this question has an affirmative answer in the more general setting where $R$ is a factorial regular ring containing $\mathbb{Q}$.

\section{The $(2, n)$-cancellation problem over Noetherian one-dimensional domains containing $\mathbb{Q}$}

Let $R$ be a ring containing $\mathbb{Q}$, and let $A$ be an $R$-algebra. It is proved in [8] that if $R$ is a Dedekind domain and $A^{[n]} \cong_{R} R^{[n+2]}$, for some $n \geq 1$, then $A \cong_{R} R^{[2]}$ (see also [12, Theorem 4.5]). In this section, we show that the same result holds true over an arbitrary Noetherian one-dimensional domain $R$ containing $\mathbb{Q}$.

\section{THEOREM 3.1}

Let $R$ be a Noetherian one-dimensional domain containing $\mathbb{Q}$. Then for every $R$-algebra $A$ such that $A^{[n]} \cong_{R} R^{[n+2]}$, for some $n \geq 1$, we have $A \cong_{R} R^{[2]}$.

\section{Proof}

Let $x=x_{1}, \ldots, x_{n}$ be a list of indeterminates over $A$, and let $y=y_{1}, \ldots, y_{n+2}$ be a list of algebraically independent elements of $A[x]$ over $R$ such that $A[x]=R[y]$.

From Theorem 2.7 we deduce that $A$ is an $\mathbb{A}^{2}$-fibration over $R$. Then by Theorem 2.6 there exists $u \in A$ such that $A$ is an $\mathbb{A}^{1}$-fibration over $R[u]$. To prove that $A$ is trivial over $R[u]$ it suffices by Corollary 2.5 to find a fixed point free $R[u]$-derivation of $A$.

Let us consider the $R[u, x]$-derivation of $A[x]=R[y]$ defined for every $f \in R[y]$ by

$$
\xi(f)=\operatorname{det} \operatorname{Jac}_{y}(u, x, f) .
$$

Let us first prove that $\xi(A) \subseteq A$. Let $K$ be the quotient field of $R$, and let $S=R \backslash\{0\}$. Since $A$ is an $\mathbb{A}^{1}$-fibration over $R[u]$ the localization $A_{S}$ is an $\mathbb{A}^{1}$ fibration over $R[u]_{S}=K[u]$. Since, moreover, $K[u]$ is a PID, $A_{S}$ is trivial over $K[u]$, and so there exists $v \in A$ such that $A_{S}=K[u][v]=K[u]^{[1]}$. This gives $A[x]_{S}=K[u, x, v]=K[y]$, and hence $u, x, v$ is a coordinate system of $K[y]$. From this fact it follows that $\xi(v) \in K \backslash\{0\}$. On the other hand, since $v \in A$ we have $\xi(v) \in A[x]$, and so $\xi(v) \in A[x] \cap(K \backslash\{0\})=R \backslash\{0\}$ by the faithful flatness of $A$ over $R$. Now, if $a \in A$ we can write $a=p(u, v)$ in $A_{S}=K[u, v]$, and applying $\xi$ to $a$ we get $\xi(a)=\partial_{v} p(u, v) \xi(v) \in A_{S} \cap A[x]=A$. Thus, if we let $\xi_{0}$ be the restriction of $\xi$ to $A$, then $\xi_{0} \in \operatorname{Der}_{R[u]}(A)$ and $\xi$ is nothing but the extension of $\xi_{0}$ to $A[x]$ obtained by letting $\xi\left(x_{i}\right)=0$. It follows that $\xi(A[x])$ and $\xi_{0}(A)$ generate the same ideal of $A[x]$, and since $A[x]$ is faithfully flat over $A$, the derivation $\xi_{0}$ is fixed point free if and only if $\xi$ is fixed point free. 
Assume towards contradiction that $\xi$ is not fixed point free. Let $\mathfrak{m}$ be a maximal ideal of $A[x]$ that contains $\xi(A[x])$, and let $\mathfrak{m}_{0}=R \cap \mathfrak{m}$. Since $\xi(v) \in$ $\xi(A[x]) \subset \mathfrak{m}$ and $\xi(v) \in R \backslash\{0\}$ we have $\mathfrak{m}_{0} \neq(0)$, and hence $\mathfrak{m}_{0}$ is a maximal ideal since $R$ is assumed to be a one-dimensional domain.

Notice that the inclusion homomorphisms $R \hookrightarrow R[u] \hookrightarrow A \hookrightarrow A[x]$ are faithfully flat. In particular, we have the following commutative diagram, where the $\pi_{i}$ 's and $\pi$ stand for the canonical projections:

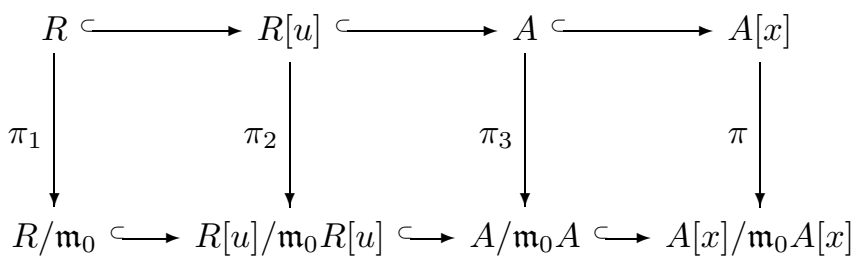

Let $\bar{\xi}$ be the derivation of $A[x] / \mathfrak{m}_{0} A[x]$ induced by $\xi$. Then we have $\bar{\xi} \circ \pi=$ $\pi \circ \xi$. On the other hand, we have

$$
A[x] / \mathfrak{m}_{0} A[x]=R[y] / \mathfrak{m}_{0} R[y] \cong_{R / \mathfrak{m}_{0}}\left(R / \mathfrak{m}_{0}\right)^{[n+2]},
$$

and $\pi(y)=\pi\left(y_{1}\right), \ldots, \pi\left(y_{n+2}\right)$ is a coordinate system of $A[x] / \mathfrak{m}_{0} A[x]$ over $R / \mathfrak{m}_{0}$. Since, moreover, $\xi=\operatorname{det} \operatorname{Jac}_{y}(u, x,-)$ the derivation $\bar{\xi}$ is nothing but the Jacobian derivation $\operatorname{det} \mathrm{Jac}_{\pi(y)}(\pi(u), \pi(x),-)$.

Now we show that $\pi(u), \pi(x)$ can be extended to a coordinate system of $A[x] / \mathfrak{m}_{0} A[x]$ over $R / \mathfrak{m}_{0}$. Since $A$ is an $\mathbb{A}^{1}$-fibration over $R[u]$, it follows that $A / \mathfrak{m}_{0} A$ is an $\mathbb{A}^{1}$-fibration over $R[u] / \mathfrak{m}_{0} R[u] \cong{ }_{R / \mathfrak{m}_{0}}\left(R / \mathfrak{m}_{0}\right)^{[1]}$. The fact that $R / \mathfrak{m}_{0}$ is a field then implies that $A / \mathfrak{m}_{0} A$ is trivial over $R[u] / \mathfrak{m}_{0} R[u]$, and so we can find $w \in A$ such that $\pi_{3}(w)$ generates $A / \mathfrak{m}_{0} A$ as an $\left(R[u] / \mathfrak{m}_{0} R[u]\right)$ algebra. As a consequence, $\pi_{3}(u), \pi_{3}(w)$ generate $A / \mathfrak{m}_{0} A \cong_{R / \mathfrak{m}_{0}}\left(R / \mathfrak{m}_{0}\right)^{[2]}$ as an $R / \mathfrak{m}_{0}$-algebra. On the other hand, we have $A[x] / \mathfrak{m}_{0} A[x] \cong_{A / \mathfrak{m}_{0} A}\left(A / \mathfrak{m}_{0} A\right)^{[n]}$, and the system $\pi(x)$ generates $A[x] / \mathfrak{m}_{0} A[x]$ over $A / \mathfrak{m}_{0} A$. It then follows that $\pi(u), \pi(w), \pi(x)$ is a generating, and hence a coordinate, system of $A[x] / \mathfrak{m}_{0} A[x]$ over $R / \mathfrak{m}_{0}$. This shows that $\bar{\xi}(\pi(w))=\pi(\xi(w))$ is a unit in $A[x] / \mathfrak{m}_{0} A[x]$, and hence there exist $\alpha \in A[x]$ and $f \in \mathfrak{m}_{0} A[x]$ such that $\alpha \xi(w)=1+f$. Since $\alpha \xi(w) \in$ $\mathfrak{m}$ and $f \in \mathfrak{m}_{0} A[x] \subseteq \mathfrak{m}$ we get $1 \in \mathfrak{m}$, which contradicts the fact that $\mathfrak{m}$ is a proper ideal of $A[x]$.

\section{REMARK 3.2}

If in addition to the assumptions of Theorem 3.1 the $\operatorname{ring} R$ is seminormal we can supply a much shorter proof. Indeed, by Theorem $2.7, A$ is an $\mathbb{A}^{2}$-fibration over $R$. On the other hand, from a corollary of Theorem 2.6, see [3, Corollary 3.9], we have $A \cong_{R} \operatorname{Sym}_{R}(M)^{[1]}$ for some finitely generated projective $R$-module $M$ of rank one. This gives $\operatorname{Sym}_{R}(M)^{[n+1]} \cong_{R} R^{[n+2]}$ and then $\operatorname{Sym}_{R}(M) \cong_{R} R^{[1]}$ by Theorem 2.3. Since $A \cong_{R} \operatorname{Sym}_{R}(M)^{[1]}$ we finally get $A \cong_{R} R^{[2]}$.

\section{REMARK 3.3}

From Theorem 2.7 it follows that Theorem 3.1 is equivalent to saying that every 
stably trivial $\mathbb{A}^{2}$-fibration over a Noetherian one-dimensional domain containing $\mathbb{Q}$ is trivial.

As a direct consequence of Theorem 3.1 we have the following result.

\section{COROLLARY 3.4}

Let $R$ be a Noetherian domain containing $\mathbb{Q}$, and let $A$ be a stably trivial $\mathbb{A}^{2}$ fibration over $R$. Then for every prime ideal $\mathfrak{p}$ of $R$ such that $R / \mathfrak{p}$ is onedimensional we have

$$
A / \mathfrak{p} A \cong_{R / \mathfrak{p}}(R / \mathfrak{p})^{[2]}
$$

\section{Proof}

Since $A$ is a stably trivial $\mathbb{A}^{2}$-fibration over $R$, it follows that $A / \mathfrak{p} A$ is a stably trivial $\mathbb{A}^{2}$-fibration over $R / \mathfrak{p}$. The claimed result then follows from Theorem 3.1 since $R / \mathfrak{p}$ is assumed to be one-dimensional.

Given a field $K$ of characteristic zero, it is still an open problem whether every $\mathbb{A}^{2}$-fibration over the polynomial ring $K^{[2]}$ is trivial. The following result gives a property of such fibrations.

\section{COROLLARY 3.5}

Let $K$ be a field of characteristic zero, and let $A$ be an $\mathbb{A}^{2}$-fibration over $R=K^{[2]}$. Then for every prime polynomial $p$ of $R$ we have

$$
A / p A \cong_{R / p R}(R / p R)^{[2]} .
$$

\section{Proof}

As noticed in the paragraph after Theorem 2.2, it follows from [2, Corollary 3.5] and the Quillen-Suslin Theorem that every $\mathbb{A}^{m}$-fibration over a polynomial ring over a field is stably trivial. Thus, $A$ is a stably trivial $\mathbb{A}^{2}$-fibration over $K^{[2]}$, and the claimed result follows from Corollary 3.4.

The above corollary answers in particular a question raised by Vénéreau (see [15, Problem 13]) concerning the polynomial $v_{1}=y+x\left[x z+y\left(y u+z^{2}\right)\right]$, a candidate counterexample to several open problems in affine algebraic geometry. More general methods to construct Vénéreau-type polynomials can be found in [7] and $[22]$.

Let $R=\mathbb{C}\left[x, v_{1}\right]=\mathbb{C}^{[2]}$, and let $A=\mathbb{C}[x, y, z, u]=\mathbb{C}^{[4]}$. It is proved in [27] that $A$ is an $\mathbb{A}^{2}$-fibration over $R$. But to our knowledge it is still an open question whether $A$ is trivial over $R$. Clearly, if for some prime polynomial $p \in R$ the fibration $A / p A$ is not trivial over $R / p R$, then $A$ is not a trivial fibration over $R$. Vénéreau's question [15, Problem 13] was then whether this is the case for some prime polynomial $p \in R$. He also proposed $p=x^{2}-v_{1}^{3}$ as an example for which no answer was known. In [13] van den Essen, Maubach and Vénéreau obtained 
that $A /\left(x^{2}-v_{1}^{3}\right) A$ is trivial over $R /\left(x^{2}-v_{1}^{3}\right) R$ as a consequence of their main result. But in fact, Corollary 3.5 shows that this holds for every prime polynomial $p \in \mathbb{C}\left[x, v_{1}\right]$.

\section{References}

[1] S. S. Abhyankar, W. Heinzer, and P. Eakin, On the uniqueness of the coefficient ring in a polynomial ring, J. Algebra 23 (1972), 310-342. MR 0306173.

[2] T. Asanuma, Polynomial fibre rings of algebras over Noetherian rings, Invent. Math. 87 (1987), 101-127. MR 0862714. DOI 10.1007/BF01389155.

[3] T. Asanuma and S. M. Bhatwadekar, Structure of $\mathbf{A}^{2}$-fibrations over one-dimensional Noetherian domains, J. Pure Appl. Algebra 115 (1997), 1-13. MR 1429296. DOI 10.1016/S0022-4049(96)00005-9.

[4] H. Bass, E. H. Connell, and D. L. Wright, Locally polynomial algebras are symmetric algebras, Invent. Math. 38 (1976/77), 279-299. MR 0432626.

[5] S. M. Bhatwadekar and A. K. Dutta, "On affine fibrations" in Commutative Algebra (Trieste, 1992), World Sci., River Edge, N. J., 1994, 1-17. MR 1421074.

[6] S. M. Bhatwadekar and A. K. Dutta, On $\mathbf{A}^{1}$-fibrations of subalgebras of polynomial algebras, Compositio Math. 95 (1995), 263-285. MR 1318088.

[7] D. Daigle and G. Freudenburg, "Families of affine fibrations" in Symmetry and Spaces, Progr. Math. 278, Birkhäuser, Boston, 2010, 35-43. MR 2562622. DOI 10.1007/978-0-8176-4875-6_3.

[8] H. Derksen, A. van den Essen, and P. van Rossum, An extension of the Miyanishi-Sugie cancellation theorem to Dedekind rings, technical report, University of Nijmegen, 2002.

[9] A. K. Dutta, On $\mathbf{A}^{1}$-bundles of affine morphisms, J. Math. Kyoto Univ. 35 (1995), 377-385. MR 1359003.

[10] A. K. Dutta and N. Onoda, Some results on codimension-one $\mathbf{A}^{1}$-fibrations J. Algebra 313 (2007), 905-921. MR 2329576.

DOI 10.1016/j.jalgebra.2006.06.040.

[11] M. El Kahoui and M. Ouali, Fixed point free locally nilpotent derivations of $\mathbb{A}^{2}$-fibrations, J. Algebra 372 (2012), 480-487. MR 2990022.

DOI 10.1016/j.jalgebra.2012.09.025.

[12] A. van den Essen, "Around the cancellation problem" in Affine Algebraic Geometry, Osaka Univ. Press, Osaka, 2007, 463-481. MR 2330485.

[13] A. van den Essen, S. Maubach, and S. Vénéreau, The special automorphism group of $R[t] /\left(t^{m}\right)\left[x_{1}, \ldots, x_{n}\right]$ and coordinates of a subring of $R[t]\left[x_{1}, \ldots, x_{n}\right]$, J. Pure Appl. Algebra 210 (2007), 141-146. MR 2311177.

DOI 10.1016/j.jpaa.2006.09.013.

[14] G. Freudenburg, Derivations of $R[X, Y, Z]$ with a slice, J. Algebra 322 (2009), 3078-3087. MR 2567411. DOI 10.1016/j.jalgebra.2008.05.007. 
[15] G. Freudenburg and P. Russell, "Open problems in affine algebraic geometry" in Affine Algebraic Geometry, Contemp. Math. 369, Amer. Math. Soc., Providence, 2005, 1-30. MR 2126651. DOI 10.1090/conm/369/06801.

[16] T. Fujita, On Zariski problem, Proc. Japan Acad. Ser. A Math. Sci. 55 (1979), 106-110. MR 0531454.

[17] E. Hamann, On the R-invariance of $R[x]$, J. Algebra 35, (1975), 1-16. MR 0404233.

[18] M. Hochster, Nonuniqueness of coefficient rings in a polynomial ring, Proc. Amer. Math. Soc. 34 (1972), 81-82. MR 0294325.

[19] T. Kambayashi, On the absence of nontrivial separable forms of the affine plane, J. Algebra 35 (1975), 449-456. MR 0369380.

[20] T. Kambayashi and D. Wright, Flat families of affine lines are affine-line bundles, Illinois J. Math. 29 (1985), 672-681. MR 0806473.

[21] H. Kraft, Challenging problems on affine n-space, Astérisque 237 (1996), 295-317, Séminaire Bourbaki 1994/95, no 802. MR 1423629.

[22] D. Lewis, Vénéreau-type polynomials as potential counterexamples, J. Pure Appl. Algebra 217 (2013), 946-957. MR 3003318.

DOI 10.1016/j.jpaa.2012.09.018.

[23] M. Miyanishi, Curves on Rational and Unirational Surfaces, Tata Inst. Fund. Res. Lectures Math. Phys. 60, Tata Inst. Fund. Res., Bombay; Narosa, New Delhi, 1978. MR 0546289.

[24] M. Miyanishi and T. Sugie, Affine surfaces containing cylinderlike open sets, J. Math. Kyoto Univ. 20 (1980), 11-42. MR 0564667.

[25] P. Russell, On affine-ruled rational surfaces, Math. Ann. 255 (1981), 287-302. MR 0615851. DOI 10.1007/BF01450704.

[26] A. Sathaye, Polynomial ring in two variables over a DVR: a criterion, Invent. Math. 74 (1983), 159-168. MR 0722731. DOI 10.1007/BF01388536.

[27] S. Vénéreau, Automorphismes et variables de l'anneau de polynomes $A\left[y_{1}, \ldots, y_{n}\right]$, Ph.D. dissertation, Université de Grenoble I, Institut Fourier, Grenoble, 2001.

El Kahoui: Department of Mathematics, Faculty of Sciences Semlalia, Cadi Ayyad University, P.O. Box 2390, Marrakesh, Morocco; elkahoui@uca.ma

Ouali: Department of Mathematics, Faculty of Sciences Semlalia, Cadi Ayyad University, P.O. Box 2390, Marrakesh, Morocco; mustouali@gmail.com 